\title{
Strategic Analysis for the MER Cape Verde Approach
}

\author{
Daniel Gaines, Paolo Belluta, Jennifer Herman, Pauline Hwang, \\ Ryan Mukai, Dan Porter, Byron Jones and Eric Wood \\ Jet Propulsion Laboratory \\ California Institute of Technology \\ 4800 Oak Grove Drive \\ Pasadena, California 91109
}

John Grotzinger, Lauren Edgar and Alex Hayes

California Institute of Technology

1200 East California Blvd

Pasadena, CA 91125

\author{
Trent Hare \\ U.S. Geological Survey \\ 2255 North Gemini Drive \\ Flagstaff, AZ 86001
}

\author{
Steve Squyres \\ Cornell University \\ 428 Space Sciences Building \\ Ithaca, NY 14853
}

\begin{abstract}
The Mars Exploration Rover Opportunity has recently completed a two year campaign studying Victoria Crater. The campaign culminated in a close approach of Cape Verde in order to acquire high resolution imagery of the exposed stratigraphy in the cliff face. The close approach to Cape Verde provided significant challenges for every subsystem of the rover as the rover needed to traverse difficult, uncharacterised terrain and approach a cliff face with the potential of blocking out solar energy and communications with Earth. In this paper we describe the strategic analyses performed by the science and engineering teams so that we could successfully achieve the science objectives while keeping the rover safe.
\end{abstract}

\section{INTRODUCTION}

The Mars Exploration Rover Opportunity has spent nearly two years exploring the bays and capes of Victoria Crater. Using a rover to explore Victoria provides an unprecedented ability to peer back in time through Martian history by studying the layered sedimentary rocks and outcrop exposed by the impact. Cape Verde (Figure 1), in particular, provides a stunning example with nearly 6 meters of exposed stratigraphy. A primary goal of the Victoria Crater campaign has been to perform a close approach to Cape Verde in order to acquire high resolution panoramic imagery of the promontory.

Cape Verde proved to be an extremely challenging scientific objective for every subsystem of the rover. A close approach of the cape required the rover to traverse unknown, treacherous terrain as it moved into the shadow of the cliff with the potential of blocking sunlight from the solar powered vehicle in the middle of winter along with the potential to block out communications with Earth preventing the team from communicating with the rover if something should go wrong. The campaign required that the team perform careful analysis of every vehicle subsystem and interactions among subsystems in order to ensure that the highest quality science was achieved while keeping the rover safe. In this paper we recount the challenges faced by each subsystem and review the

978-1-4244-2622-5/09/\$25.00 (C)2009 IEEE.
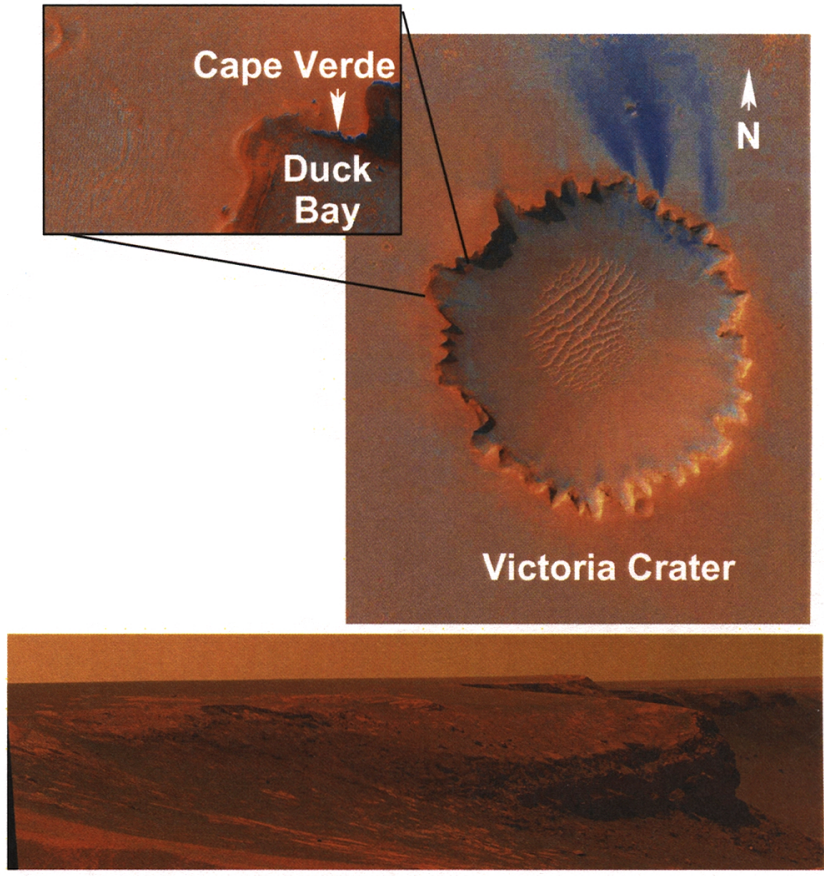

Figure 1. Cape Verde (images from MRO/HiRISE and Opportunity Rover).

analysis that was performed in support of the approach.

The most immediate challenge faced in the Cape Verde campaign was getting the vehicle close to the cape without getting trapped in the uncharacterized soil and slopes along the way. We describe the traversability analysis that was performed in order to identify a safe route to the cape.

One of the most sobering prospects of the operation was the fact that we were moving a solar powered rover toward the shadow of a south-facing cliff in the middle of winter with the sun low in the Northern sky. Power models that take into account occlusions of the sun from terrain had never been developed for the mission as no one had considered taking a solar powered vehicle close to a cliff. A miscalculation here 


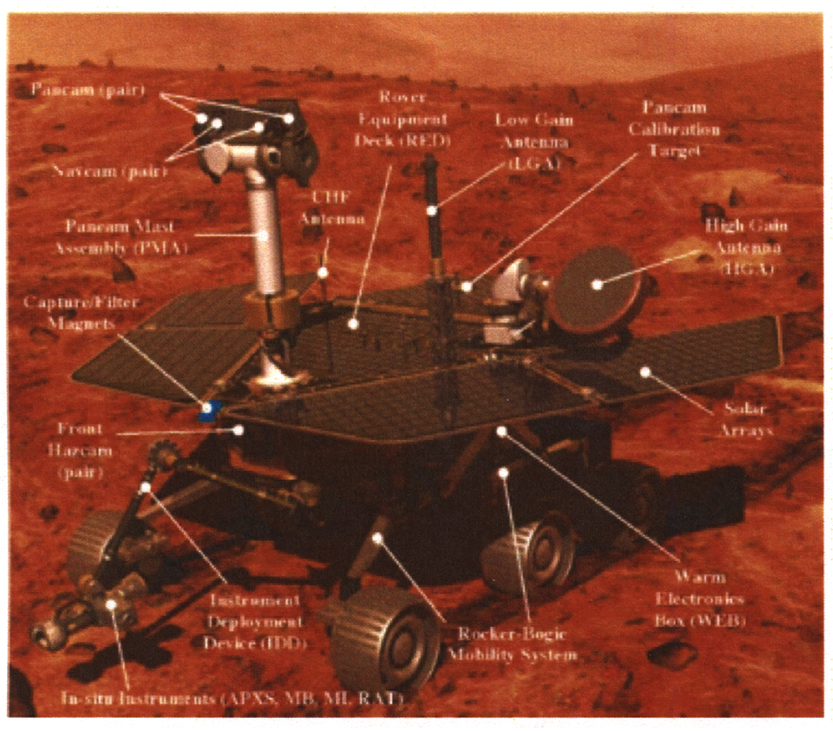

Figure 2. A Mars Exploration Rover.

could result in leaving the rover stranded in a location where it could not take in enough solar energy to heat itself through the night which could result in loss of the spacecraft. We describe how a new power model was developed to enable the team to make decisions about where we could take the rover. Additional analyses were performed on the thermal subsystem to determine how much additional system heating may be required if the rover moved into shadows.

Occlusions of the sun were not the only dangers posed by the cape wall. The wall also had the potential to block Earth as the team tries to upload new commands to the rover, and to block the rover's view of the Odyssey orbiter as it tries to send data to Odyssey to downlink to Earth. Analyses were performed to assess the impact the cape would have on the telecommunications subsystem.

The following describes in more detail the analyses that were performed by each subsystem to ensure the success of the mission and safety of the rover.

\section{SCIENCE OBJECTIVES AND ANALYSIS}

The primary goal of the Cape Verde imaging campaign was to correlate the stratigraphy at the Duck Bay ingress section with the stratigraphy observed in cliff faces during the traverse of Victoria Crater's rim. While other cliff faces were observed at typical ranges of $60 \mathrm{~m}$ or greater, the Opportunity rover was able to navigate to a stand-off distance of $\sim 10$ $\mathrm{m}$ from Cape Verde, providing native resolutions of up to 3 $\mathrm{mm} /$ pixel. In addition to standard image acquisition, superresolution sequences were employed on five high-priority science targets that were identified based on a previous imaging of Cape Verde from sol 1342 (Figure 3). Super-Resolution sequences combine information from multiple images taken from slightly different pointing geometries in order to extrap- olate a new image at higher than native resolution [1].

Each of the five super-resolution targets were chosen to answer a specific questions regarding the unique nature of the bedding at Cape Verde (see Figures $3 \& 16$ ). Target A was intended to analyze a distinct boundary that appeared to be an erosional contact at the base of the cliff face. Target B aimed to understand dark and light bands (which appeared to be multiple diagenetic horizons) superimposed on bedding. Target $\mathrm{C}$ consisted of a unique erosional ridge, also superimposed on bedding. Target $\mathrm{D}$ was intended to capture what appeared to be grainflow strata transitioning into finer scale bedding below a possible contact. Target $\mathrm{E}$ was chosen to study a possible erosional contact or set boundary at the base of the cliff face, likely a longer exposure of the same surface as seen in Target A.

Cape Verde is an East-West trending promontory with a vertical exposure of $\sim 7 \mathrm{~m}$, length of $\sim 25 \mathrm{~m}$, and average slope of $\sim 0^{\circ}$ (see Figure 3). The stand-off distance to the Cape was chosen based on safety constraints and a desire to keep the camera bore-sight within $45^{\circ}$ of the cliff's surface normal. Time of day was critical to successful imaging as solar illumination originated from the northern portion of the sky and commonly resulted in glinting off facets in the cliff face. This problem was exacerbated by the rover's proximity to Cape Verde, requiring the camera mast to angle up towards the sky in order to keep the cliff in its field-of-view. Optimal lighting under these conditions consisted of diffuse illumination during times when the solar incidence angle was far enough away from the angle between the target's surface normal and the camera bore-sight.

\section{Mobility ANAlysis}

The first challenge we faced in the Cape Verde approach campaign was identifying a safe path the rover could follow across Duck Bay to get close to the cape. Details on the MER mobility system and an overview of mobility operations with Opportunity can be found in [2], [3], [4]. The general approach we took to the Cape Verde approach analysis was to first identify the closest point we could safely get the rover to Cape Verde considering traversability analysis alone. We would then use the path to get to that point as a basis for the analysis of the other subsystems. If necessary, we could back off from that closest point if it turned out it would not be safe from some subsystem perspective.

The highest concern in the traversability analysis was to avoid getting the rover stuck. Getting close to the cape was a major science objective, but we did not want to pay the price of not being able to get back out of the crater and continue exploration. The major criteria in finding a safe path were to take advantage of outcrop when possible, avoid areas that appeared to consist of deep, loose soil and favor paths that minimized the slopes the rover encountered. We also wanted to delay as long as feasible putting the rover on slopes that would tilt it away from the sun so that we could maximize the 


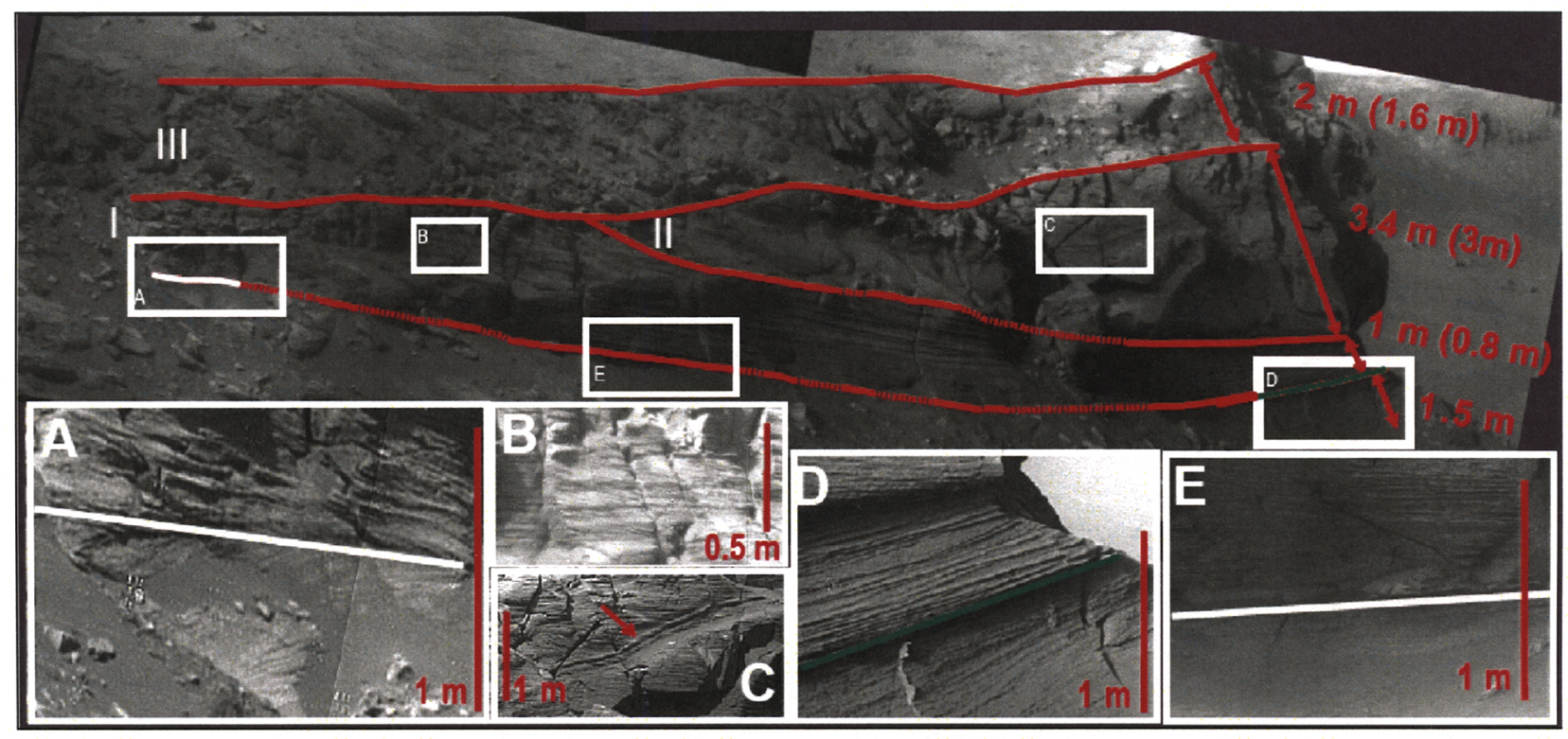

Figure 3. Cape Verde cliff face as observed on MERB sol 1371. Targets A through E represent high priority science targets that were imaged using super-resolution techniques. Please see text for a description of each target. Labels I, II, and III distinguish large-scale variations in observed stratigraphy; I. Thickly bedded dune toeset laminae (grainflow) II. Dune foreset cross-stratifications formed of climbing translatent strata with evidence for superimposed wind ripples. III. Impact breccia.

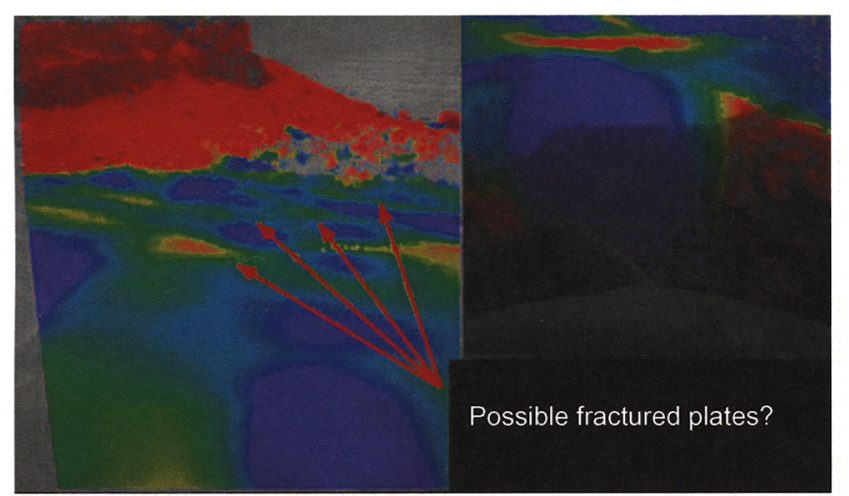

Figure 4. Slope analysis of terrain leading to Cape Verde: blue indicates slopes $<15^{\circ}$, red indicates slopes $>20^{\circ}$.

solar input to the rover during the campaign.

Much of the traversability analysis was based on slope maps which are derived from the stereo images acquired by the rover's cameras. Figure 4 shows an example slope map of the area in from of Cape Verde. Colors are used to indicate the severity of the slopes. In this map, blue is used for slopes less than $15^{\circ}$ and red for slopes greater than $20^{\circ}$. Note that close to the cape face the slopes start to increase rapidly. These slopes are also facing South, so that when the rover encounters them it would be tilted away from the sun, which in the Winter season for Opportunity was in the North.

Two key locations were identified for the campaign and are shown in Figure 5. The location labeled "Goal" was identi-

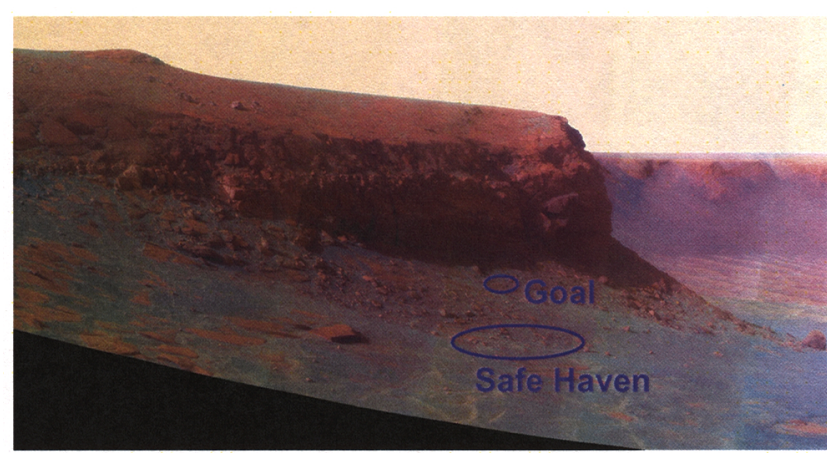

Figure 5. Key locations in analysis.

fied as the closest point we could safely get the rover taking into account traversability concerns alone. "Safe Haven" was selected as a sort of "base camp" for the final approach. This is the last area where the rover would have favorable tilts relative to the sun before climbing up the steeper, South-facing slope of the cape face. It was also our retreat location in case our power predictions were not on target and we found ourselves with too little power. We could quickly retreat back to Safe Haven and recharge the vehicle.

At the time we began this analysis, we had been exploring the interior of Cape Verde for several months and had learned some of the challenges and surprises the terrain held. The most important discovery for traversability analysis was that the terrain leading toward Cape Verde had a series of steplike features resulting in relatively sharp drops in the slope. These "steps" show up as small red regions in the slope map 


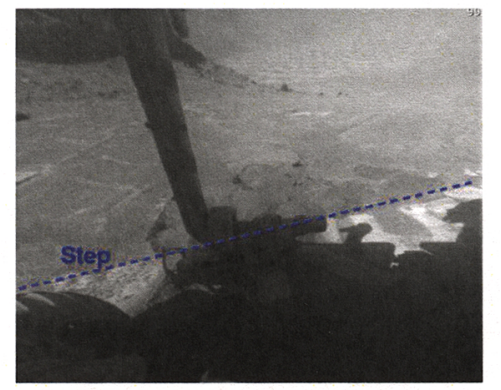

Figure 6. Sol 1557 front hazcam showing a step feature.

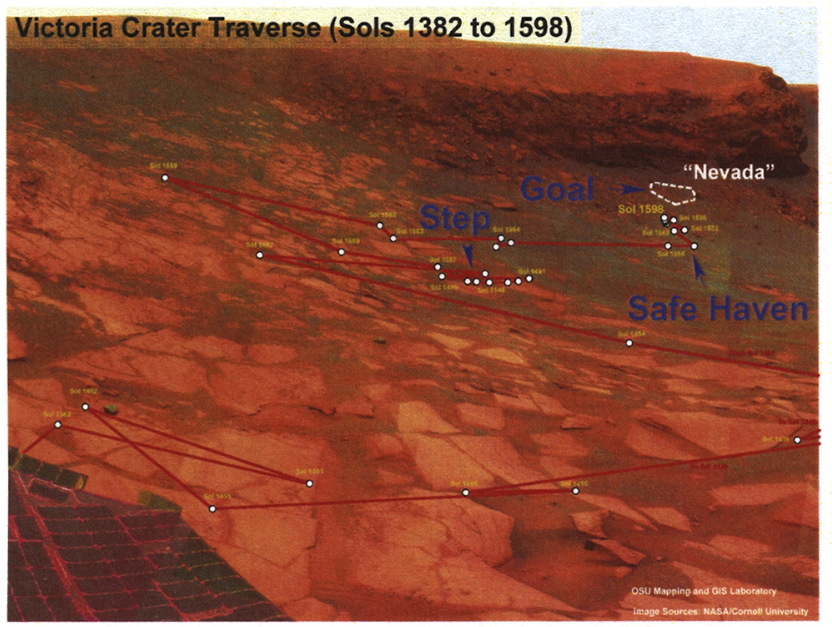

Figure 7. Actual path taken to approach Cape Verde.

of Figure 4. One hypothesis to explain these features is that this area of Duck Bay consists of a series of fractured plates and the steps are the transition from one plate to the next with sand filling in the gap. In fact, we had a difficult time with one of these steps, taking several sols to extract the rover over the lip. Figure 6 is a front hazcam image taken on Sol 1557 after the rover successfully backed up over a step.

The final path we used is shown in Figure 7. After extracting the rover from the step on sol 1557, we deviated from the originally planned path and went back up-slope and toward the cape. This allowed us to take a route that avoided the larger of the step features that we saw in Figure 4.

\section{HORIZON MASKS}

A topographic map of Victoria Crater was created from a pair of images collected by the Mars Reconnaissance Orbiters High Resolution Imaging Science Experiment (HiRISE) instrument [5], [6]. Using this topographic data, a series of horizon masks were generated. A horizon mask is a 360 degree sweep in azimuth, from a single location, showing the vertical angle to the horizon (intersection of surface and sky). For example, Figure 8 contains the horizon mask centered at Safe Haven and positioned 1 meter off the ground (the height of Opportunitys solar panels). The $\mathrm{X}$-axis in the graph repre-

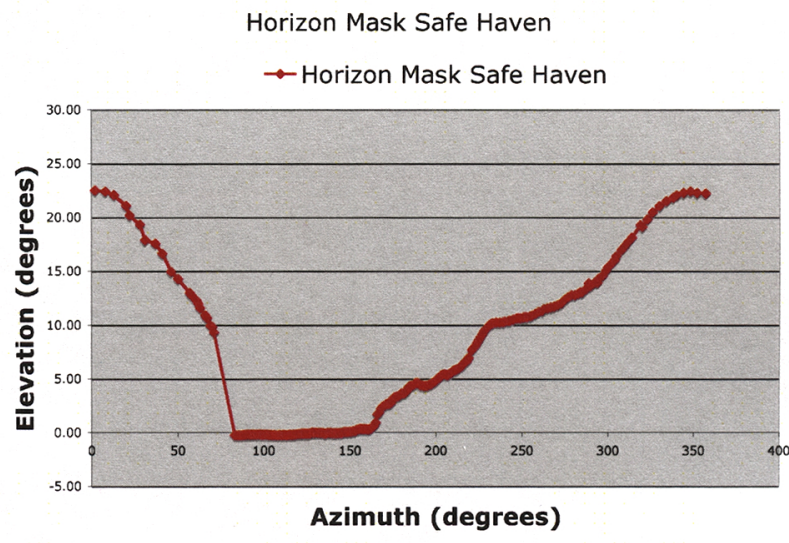

Figure 8. Horizon mask at Safe Haven (data obtained from MRO/HiRISE and USGS).

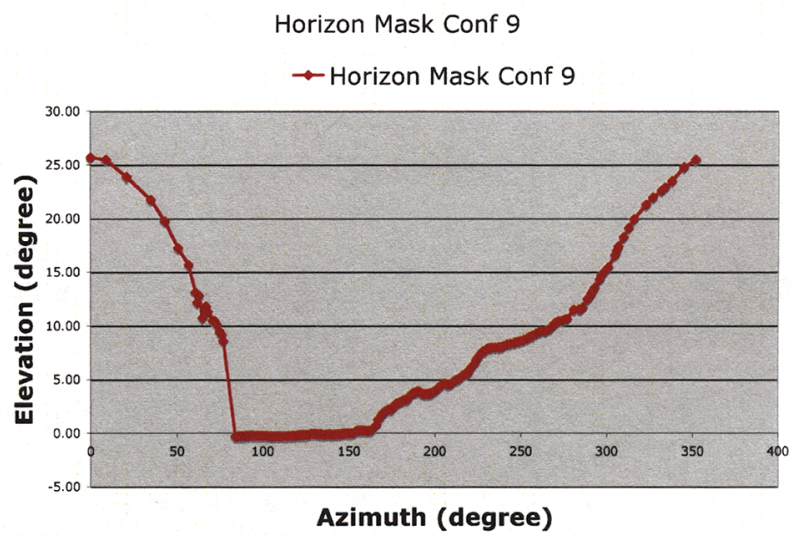

Figure 9. Horizon mask at Goal location (data obtained from MRO/HiRISE and USGS).

sents azimuth with 0 directly north and 90 directly east. The Y-axis corresponds to the vertical angle which intersects the horizon in that azimuth direction. Figure 8 indicates that, at Safe Haven, the terrain directly north of the Rovers deck (i.e. toward the cape face) is at an angle of about $23^{\circ}$.

For comparison, the horizon mask for the Goal location is in Figure 9. Here, the elevation due North is higher, with an elevation of about $25^{\circ}$. This of course make sense as the Goal location is closer to the cape face which lies to the North of the rover. In contrast, the elevations in the horizon mask in the East, South and West do not change as much between these two locations.

The horizon mask played an important role in many of the analyses we will see in the upcoming sections. For example, knowing the elevation of the horizon at a given location allowed the power engineer to determine how much the terrain will block out solar energy. 


\section{Power Analysis}

The Mars Exploration Rovers are powered by solar panels and two rechargeable Lithium-ion batteries which store up power when sunlight is not available or the power draw on the rover exceeds the solar input. The batteries are charged in turn when the solar input to the solar panels exceeds the power current needs of the vehicle.

Like Earth, Mars is axially tilted and, as such, the amount of solar energy varies with the seasons. Situated to the South of the equator, Opportunity and Spirit experience winter when the sun is to the North. Opportunity is near the equator so it does not have the larger fluctuations in power experienced by the Spirit rover position further South, but the variance between winter and summer can still be significant for the rover.

We had originally planned to complete the exploration of Duck Bay, including the approach of Cape Verde well before Winter Solstice on Mars when the sun is lowest in the sky and the days are shortest. However, we ended up spending extra time exploring the crater, including responding to the loss of one of the actuators in Opportunity's arm, that the actual approach to Cape Verde was to happen exactly at Winter Solstice.

To make the campaign more challenging from the power subsystem perspective, Cape Verde is a South facing cape and would potentially block out significant amounts of what little winter solar energy there was. If power levels go too low, there would be insufficient energy in the batteries to allow the rover to run heaters during the night that are critical for its survival. Thus, we needed to be extremely careful in our modeling of predicted power to ensure that we would not take the vehicle into an area where it could not get sufficient energy to stay alive.

Going into the Cape Verde analysis, the mission already had sophisticated power models that provide high-fidelity predictions of the amount of power expected for future sols taking into account a vast array of information including the time of year, the tilt of the rover, measures of the amount of dust in the atmosphere, estimates of the amount of dust on the solar panels, expected temperatures throughout the day and night, and the starting state of the batteries and the planned activities on the rover. The operations team uses these power predicts every day to evaluate the developing command sequences and verify that the planned activities are compatible with the power subsystem.

As sophisticated as the power models were, they did not take into account loss of solar energy due to varying terrain as no one had ever seriously considered driving a solar powered vehicle into the shadow of a cliff. Thus, as part of the Cape Verde analysis, a revised power model was developed that takes into account terrain occlusions.
The power model breaks the solar input into three types of solar energy: direct, direct diffuse and isotropic. Direct solar energy accounts for the majority of the solar input and is modeled as a ray from the sun to the vehicle. The horizon masks from Section 4 along with ephemeris data to predict the azimuth and elevation of the sun over the course of a Martian day were used to determine when the terrain of the crater would occlude the direct energy of the sun. This type of occlusion corresponds to the rover being in shadow. Direct diffuse energy results from the energy from the sun being scattered by the atmosphere. It is modeled as a cylinder from the sun to the rover. As the sun changes elevation in the sky, it cuts through different amounts of atmosphere and, as a result, the diameter of the cylinder changes. Thus, the new power model tracks the diameter of the cylinder over time and again consults the horizon masks to determine how much of the cylinder gets occluded by the crater terrain. Finally, isotropic energy is energy reflected from surrounding terrain. The presence of the South facing Cape Verde while the sun is in the North means a reduction in the amount of isotropic energy from that direction.

With the revised power model, we performed a series of predictions using the horizon masks from different locations in the path from Safe Haven toward the cape face. At each location, we projected forward in time through the sols that would be required for the rover to approach the cape and return, adding in padding to account for unexpected issues. We were originally hoping to take the vehicle within 2 meters of the cape face, but our power predictions indicated that we would likely not have sufficient power during middle of winter to support such a close approach. However, the models indicated that an approach of about 4 meters would be possible.

Of course, before basing our final decisions on these brand new power models, we wanted to validate them with empirical data from Mars. Thus, during the implementation of the Cape Verde approach, we used the predictions of the power models as a guide for day to day planning and proceeded conservatively. One strategy that was used was to make use of autonomous behavior of the mobility system to stop the drive if the tilt of the rover exceeded a certain limit or if the vehicle progressed within a certain distance of the cape face. We set these limits to be sure that we kept the rover in locations where we knew it would have sufficient power even with the loss of solar energy due to the cape face. We could then look at the data that came from the rover the following day and compare it with the energy levels predicted by the new power models.

\section{Thermal ANALYSIS}

One of the many issues that had to be addressed before going into Victoria crater was the effect that being in the crater and approaching the cliffs at Cape Verde, which would place us in the shade of the cliffs for a rather lengthy time of each Sol, would have on the temperatures of the components. In order to determine the effect it was necessary to exercise the Rover 
Thermal Math Model (TMM) with conditions that would be representative of those to be encountered in the crater.

The major concern was the internal electronic components. They have very narrow Allowable Flight Temperature (AFT) limits. Most of the electronics have minimum AFT limits of about $-40^{\circ} \mathrm{C}$. There was no concern for the external hardware since they had rather wide AFT limits. The majority of the external hardware has minimum AFT limits of $-105^{\circ} \mathrm{C}$ and none of the components have shown that they were near this temperature in the past while going through the Martian winters except for the Solar Panels.

To exercise the TMM model and have it be representative of a worst case condition a sol was chosen that had low power dissipation for the activities that it was to perform throughout that sol. When the rovers drive in a given sol the drive control boards in the electronics dissipate much greater power than when science is performed. An attempt to find a sol that would have low power dissipation was desired. The one that was chosen had a Mossbauer spectrometer integration in it and therefore the Rover could actually be napping through most of the sol. And finally we performed a worst case analysis by modeling as if there were no Sun so that there was no solar insolation on the rover.

Using the power dissipation profile from the model sol the TMM was run four times in an attempt to achieve a quasi steady-state condition. The final temperatures from each run were made the initial temperatures for each succeeding run and four runs made. The results turned out to be rather benign and indicated that there was no item that would experience any temperature close to its minimum AFT limit. Table 1 shows the nominal minimum temperatures for the model sol and the resulting temperatures for the runs that were made with no solar input to the model.

\section{TELECOMMUNICATIONS ANALYSIS}

The objective of the telecommunications analysis is to ensure that we have reliable communications with the rover during the Cape Verde approach. There are two modes of communication between the rover and Earth [7]. We have a direct line of communication with the rover by transmitting between the Deep Space Network and the rover's High Gain Antenna (HGA) or Low Gain Antenna (LGA) (Figure 2). This is typically referred as an X-band pass as it uses frequencies in the $\mathrm{X}$-band range to transmit data. The use of the HGA is preferred over the LGA as it provides higher data transfer rates.

We can also communicate indirectly with the rover using a relay through the Odyssey orbiter. In these cases, when the rover sends data to Earth, it first sends the data to Odyssey, which in turn, sends the data to the Deep Station Network on Earth. During our extended missions, the nominal communication path is to use X-band to send command sequences to the rover and Odyssey relay to send data from the rover back to Earth.
In order to ensure reliable communications, we needed to analyze the impact of the approach to the cape on both X-band and Odyssey relay communications.

\section{$X$-Band Communication}

For reliable X-band communications, the rover needs an unobstructed line between Earth and the HGA of the rover (see Figure 2) during the time of the pass. There are three objects that could occlude the rover's view of Earth: 1) the rover's Pancam Mast Assembly (PMA), 2) the rover's solar panels, and 3) the terrain.

The PMA, as shown in Figure 2, includes a masthead housing the panoramic and navigation cameras. It is possible that the masthead can be positioned in such a way that it could block the line between Earth and the HGA. Fortunately, the HGA can actuate and, in these situations, the rover can re-position the antenna to avoid the PMA occlusion. Onboard software handles these cases automatically.

Depending on the tilt of the rover and the location of Earth in the Martian sky, the rover deck can also act as an occlusion during an X-band pass. The position of the Earth in the Martian sky depends on the time of year and time of day. Thus, we had to check for occlusions for the range of sols in which we would be conducting the approach and at the times of day we would be attempting to communicate with the rover. X-band passes are usually scheduled in the late morning but, depending on Deep Space Network scheduling, can occur earlier or later.

We have a tool called TBall which helps us check for these PMA and rover deck occlusions. TBall allows the user to specify a sol and time of day on that sol along with a rover attitude (roll, pitch and yaw). It then plots the plane representing the rover deck relative to a flat horizon. It also shows the position of the Earth relative to the rover deck and plots the path the Earth will follow. It is then a simple matter of determining at what time the path of the Earth crosses below the plane of the rover deck. An example TBall plot is show in Figure 10. This is for the X-band pass on sol 1570. This was a relatively late pass starting at 12:45. By the time the pass is over, at 13:05, the Earth is still well above the rover deck.

In preparing for the Cape Verde approach, we ran TBall for several uplink windows within the range of sols in which we would be performing the approach. In particular, we made sure we picked sols when we had X-band passes later in the day, as the Earth would be lower in the horizon at those times. We used the rover's attitude for the Goal configuration as this had the highest amount of tilt, and thus, the greatest chance of the deck interfering with communications. Fortunately, we found that the deck would not be in danger of occluding Earth during our planned X-band passes.

The final analysis with respect to X-band communication was to determine if the cape itself would occlude Earth during 


\begin{tabular}{|l|c|c|c|}
\hline Component & Initial Temperature ${ }^{\circ} \mathrm{C}$ & No-Sun Temperature ${ }^{\circ} \mathrm{C}$ & Delta Temperature ${ }^{\circ} \mathrm{C}$ \\
\hline \hline Battery & -16 & -20 & -4 \\
\hline SSPA & -28 & -35 & -7 \\
\hline SDST & -28 & -35 & -7 \\
\hline UHF & -28 & -34 & -6 \\
\hline REM & -27 & -34 & -7 \\
\hline MTest & -60 & -65 & -5 \\
\hline \multicolumn{3}{|c|}{} & SSPA = Solid State Power Amplifier \\
& SDST =Small Deep Space Transponder \\
& UHF =Ultra High Frequency Electronics \\
& REM =Rover Electronics Module \\
& MTest=Mini Test Electronics
\end{tabular}

Table 1. Conservative thermal modeling for Cape Verde approach.

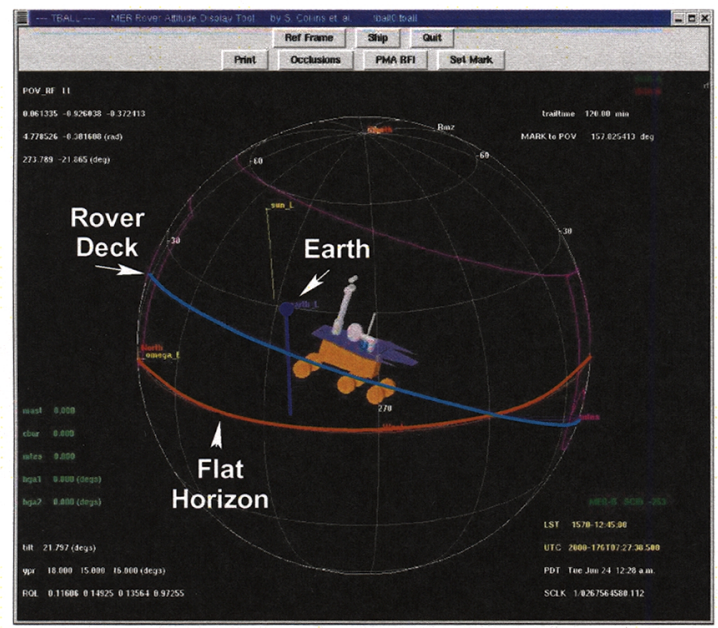

Figure 10. Using the TBall tool to check for rover deck occlusions for X-band pass on Sol 1570.

an X-band pass. Again, we looked at several of the X-band passes that would occur during the sols of the approach to see how the Earth trended toward the terrain. We used ephemeris data to predict the azimuth and elevation of the Earth with respect to the rover in the mid-morning (the earliest time for an X-band pass) and mid-afternoon (the latest time for an Xband pass). We compared the Earth azimuth and elevation with the elevation of the terrain at that azimuth as reported in the horizon masks (Section 4). We found cases where, if there were a mid-afternoon X-band pass, we would be in risk of the terrain occluding Earth, but fortunately, we did not have $\mathrm{X}$-band passes during those times.

In the end, it was determined that neither the tilt of the vehicle nor the proximity to the cape would pose a problem for our $\mathrm{X}$-band communications.

\section{Odyssey Communication}

Both the Opportunity and Spirit rovers use the Odyssey (ODY) orbiter regularly for returning both rover engineering

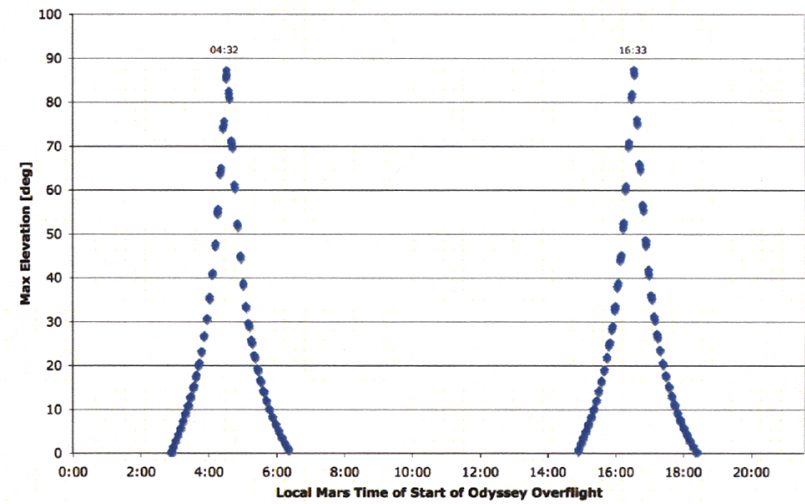

Figure 11. Distribution of Odyssey overflights times and corresponding maximum elevations.

and science data. Using the UHF link instead of X-band is less power intensive for the rover and yields much higher data returns because the Orbiters are able to continually track and downlink data to Earth at higher return data rates. Over $90 \%$ of MER rover data has been sent through Odyssey. Odyssey is considered the prime nominal downlink source for planning in the MER extended mission.

The rover can see the Odyssey orbiter on an average of 4 times per sol, the orbiter passes are distributed between midnight and sunrise (a.k.a. AM passes), and from midday to late afternoon (a.k.a. PM Passes). The project generally only uses ODY overflights that are above $10^{\circ}$ in max elevation. Passes above $10^{\circ}$ in max elevation can range in duration from 10 to 15 minutes, and the return link data rate from the rover to both orbiters can be up to $256 \mathrm{kbits} / \mathrm{s}$ but are usually $128 \mathrm{kbits} / \mathrm{s}$. Figure 11 shows a typical distribution of passes for Odyssey in local solar time units and the corresponding maximum elevation of each pass. Since the Odyssey spacecraft is a low altitude polar orbit around Mars, it rises from the North and sets in the South for one set of overflights then approximately 12 hours later when Mars has rotated $180^{\circ}$ underneath the orbit, the spacecraft rises from the South and sets in the North. 


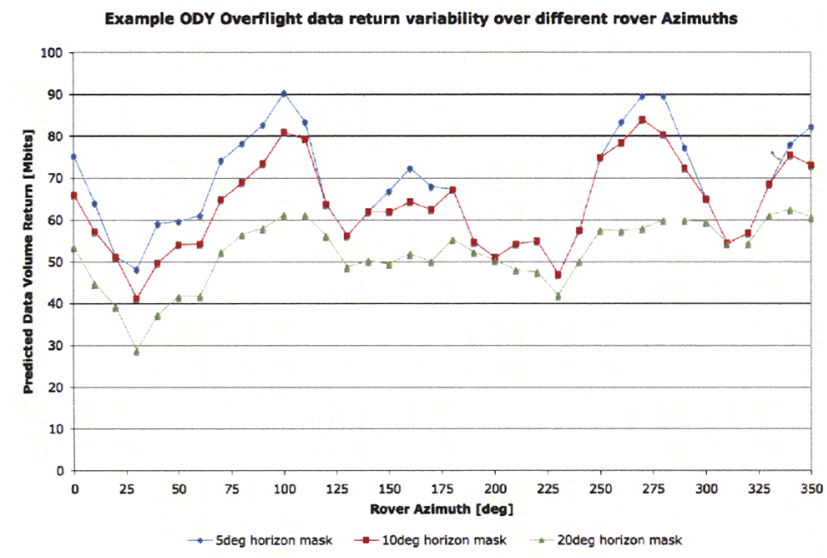

Figure 12. Predicted UHF pass volume versus rover azimuth for 3 different horizons masks applied.

Rover azimuth strongly affects link performance. Figure 12 provides an example of Odyssey overflights variability of the data volume returned showing the effects of various rover azimuths. The analysis was done using 3 basic horizon masks, $5^{\circ}, 10^{\circ}$, and $20^{\circ}$ elevation horizon masks. These are simple masks that assume a flat terrain. Even though we are able to apply these horizon masks easily to predict data volumes, we were not able to apply the same global horizon mask while inside Victoria crater and for the approach to Cape Verde because the terrain was highly variable and required a higher fidelity horizon mask.

Using the custom Cape Verde horizon masks obtained using MRO orbiter data along with the general ODY overflight geometry files delivered by the MER Navigation and Strategic teams, we were then able to merge all the provided data together using a MER tool to generate plots for analysis. Figures $13-14$ are examples of the plots for Sols 1571 and 1574 , respectively. These plots have azimuth angles along the X-axis and elevation angle of the terrain or the Odyssey spacecraft along the Y-axis. The horizon mask used for the figures is for the Safe Haven location. The red curve is the same as the curve shown in Figure 8. Each plot has the available Odyssey UHF passes that are above $10^{\circ}$ in elevation per Sol, labeled with an identification number used by the MER project ground and flight software (i.e. 45740, 45743). The first number, 4, of the 5-digit id is for Odyssey UHF windows only, the next three digits represent Sol number and the last digit is for a nighttime or daytime UHF pass. If the last digit is even then its a nighttime pass and if the last digit is odd, it is a daytime pass. The difference between the sols is the overall overflight geometry. Sol 1574 has two higher elevation passes available while Sol 1571 has 3 lower elevation passes.

Looking at Sol 1574, because both UHF passes have high Odyssey elevation angles, the passes are only obstructed by terrain either at the very beginning or at the very end of the pass by a few minutes. However, for Sol 1571, the lower elevation pass, the 45713 line, is almost completely obstructed

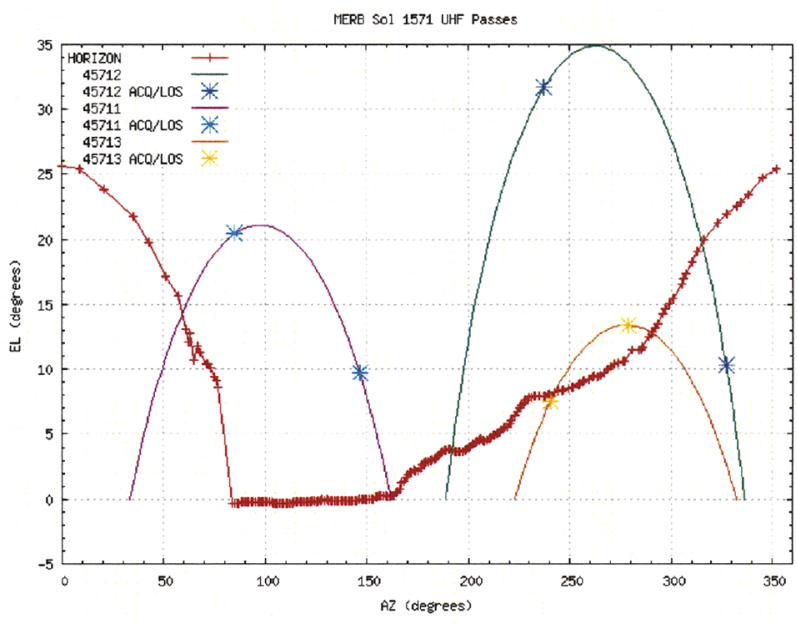

Figure 13. Sol 1571 Odyssey overflight geometry with terrain mask for Safe Haven.

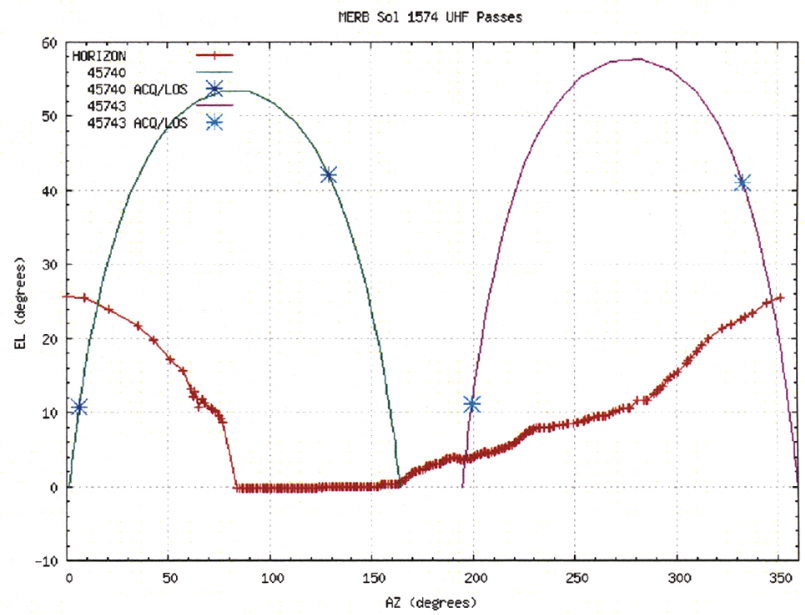

Figure 14. Sol 1574 Odyssey overflight geometry with terrain mask for Safe Haven.

by the terrain. In those cases, we would use the other UHF pass shown, in this case, 45711 (which is the other daytime UHF pass), which is only obstructed at the beginning of the pass. In general the MER ground process picked the higher elevation of the two passes, so this analysis only confirmed that the strategy did not need to change.

Figure 15 illustrates a case where choosing the higher elevation out of the two daytime passes was not optimal. In this case, the higher elevation pass, 45673 is more occluded by the terrain than the lower elevation pass, 45671. In general, this geometry occurred approximately once in every 20 sols, so it would not be a significant impact to rover operations near Cape Verde. 




Figure 15. Sol 1567 Odyssey overflight geometry with terrain mask for Safe Haven.

\section{RESUlts}

Figure 7 shows the path the rover took to reach its closest point of approach to Cape Verde. As can be seen in the figure, we did not quite get to our intended goal location. Traverses on the steep terrain just South of the goal proved to be extremely challenging. Rover plans had to be crafted very carefully to deal with the high degree of slippage and to avoid running into rocks in the area.

We debated whether or not to re-evaluate our goal location and even considered halting our approach at this location when, on sol 1600, the rover experienced an anomaly with the drive motor on its left front wheel. An unusual current spike was detected by onboard software and the drive was stopped early. The data looked eerily similar to the data observed on the Spirit rover soon before Spirit lost the drive motor on its right front wheel. This caused heightened concern that Opportunity might soon lose its left front drive motor. We had little hope that we could get Opportunity out of Victoria with only 5 functional wheels. As a result, it was decided that it was time to leave the crater and we began the process of driving Opportunity back out of Duck Bay.

Despite not reaching the original goal location, the Cape Verde approach was able to achieve our science objectives. The imaging campaign successfully captured all of the features of interest in the cliff face. A mosaic taken at dusk using the L1 filter (sol 1580) produced a very useful mosaic of the entire promontory. This mosaic revealed that Cape Verde primarily consists of low angle cross-bedding, with a light-toned diagenetic band crosscutting the physical stratigraphy. At the base of the cliff there is an erosional surface that may help to correlate the physical stratigraphy exposed at Cape Verde with stratigraphy seen in the ingress path, and elsewhere in the crater. The east side of the promontory produces a different geometry, revealing trough-like cross-bedding similar to that seen at Cape St. Mary.

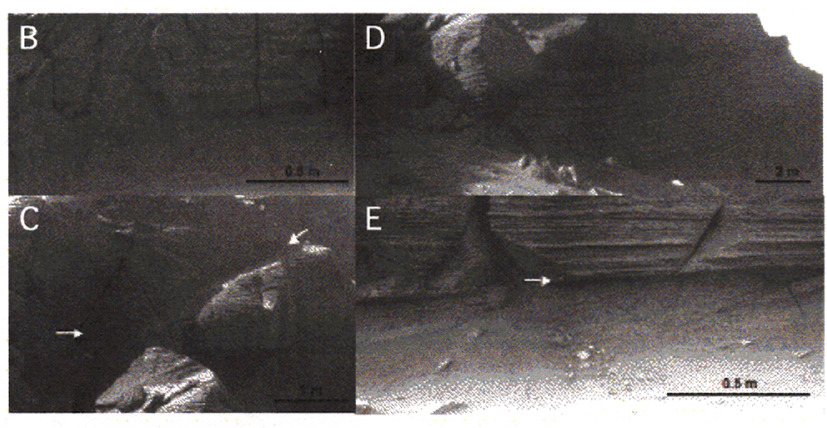

Figure 16. Cape Verde super-resolution science targets. A; Target A (not pictured) was not well exposed in the sol 1575 viewing geometry, but appeared to expose the erosional contact at the base of the Cape from the sol 1342 viewing geometry (Figure 3). B; Target B consists of light and dark bands that at first appeared to be possible multiple diagenetic horizons. However, the super-resolution image suggests that there are not multiple diagenetic horizons, and the bands are simply the result of variations in basaltic sand content, and the weathering style. $\mathrm{C}$; Target $\mathrm{C}$ was selected to study the erosional ridge (white arrows). It appears that the ridge might be the result of wind abrasion, as sand built up on the ledge below, and the area just above the ledge was preferentially eroded (the ridge parallels the ledge). D; Initial images of Target D suggested that perhaps there was evidence for grainflow, but the super-resolution images from sol 1575 suggest that this target exposes an oblique cut through a large dune. E; Target E clearly captured the erosional surface at the base of Cape Verde. This surface holds high scientific value in trying to correlate the stratigraphy in the ingress path with the stratigraphy exposed at Cape Verde, and other promontories.

In addition to the L1 panorama, super-resolution images of Targets A, E and D provided useful information regarding the erosional surface at the base of Cape Verde, and Targets B and $\mathrm{C}$ investigated possible diagenetic horizons and ridges (Figure 16). The surface at the base of the cliff face (Targets A, D, E) is clearly an erosional surface, separating low-angle crossbeds in the cliff face from higher angle bedding exposed in the scree slope below the cliff. What initially appeared to be evidence for grainflow on the east side of the promontory (Target D), can actually be explained by a change in the cliff face geometry, producing the change to trough-like crossbedding. Additional clarification was reached regarding the possible multiple diagenetic bands (Target B), which can be explained by variations in the basaltic sand content of individual beds. Details revealed in super-resolution images suggest that the erosional ridge (Target $\mathrm{C}$ ), might be the result of wind abrasion through preferential erosion, as sand built up on the ledge below the current ridge. These conclusions would not have been possible without the additional detail provide by super-resolution imaging. Additional details are provided in Figure 16.

In addition to improving our geologic understanding of the Victoria crater stratigraphy, this campaign led to improve- 
ments in processing super-resolution images and imaging under difficult lighting geometries. Even after four years on Mars, operating the MER spacecraft is still a learning process. Difficult lighting geometries associated with trying to image a south-facing cliff during southern hemisphere winter, when the sun is mostly behind the cliff face, resulted in initial versions of the super-resolution images producing artifacts such as hot pixels, large lighting gradients, and cat scratches (faint white trails visible in some of the images). Modifications of the super-resolution algorithms have been created to reduce the magnitude of these effects and will be used to process both past and future super-resolution imaging sequences.

\section{Conclusions}

The Cape Verde approach campaign proved to be an exciting and challenging operation that resulted in the collection of highly valuable science data. The operation required strategic analysis across every rover subsystem to ensure that high quality data could be collected while ensuring the safety of the spacecraft.

At the time of writing it has been 90 sols since we observed the spike on Opportunity's left front drive motor and fortunately the motor has performed nominally. Opportunity is now out of the crater and embarking on a $12 \mathrm{~km}$ trek to Endeavour Crater. The journey to Endeavor and the crater itself promise even more exciting science and engineering opportunities and challenges.

\section{ACKNOWLEDGMENTS}

Significant portions of the work described in this article were performed at the Jet Propulsion Laboratory, California Institute of Technology, under a contract with the National Aeronautics and Space Administration (NASA).

\section{REFERENCES}

[1] J. F. Bell, J. Joseph, J. N. Sohl-Dickstein, H. M. Arneson, M. J. Johnson, M. T. Lemmon, and D. Savransky, "In-flight calibration and performance of the Mars Exploration Rover Panoramic Camera (Pancam) instruments," Journal of Geophysical Review, vol. 111, 2006.

[2] J. Biesiadicki and M. Maimone, "The Mars Exploration Rover surface mobility flight software: Driving ambition," in Proceedings of the IEEE Aerospace Conference, Big Sky, Montana, March 2005.

[3] R. G. B. Jeffrey J. Biesiadecki, Eric T. Baumgartner, B. K. Cooper, F. R. Hartman, P. C. Leger, M. W. Maimone, S. A. Maxwell, A. Trebi-Ollenu, E. W. Tunstel, , and J. R. Wright, "Mars Exploration Rover surface operations: Driving opportunity at Meridiani Planum," in Proceedings of IEEE Conference on Systems, Man and Cybernetics, Hawaii, October 2005.

[4] Y. Cheng, M. Maimone, and L. Matthies, "Visual odometry on the Mars Exploration Rovers," in Proceedings of IEEE Conference on Systems, Man and Cybernetics, Hawaii, October 2005.

[5] A. McEwen, E. Eliason, J. Bergstrom, N. Bridges, C. Hansen, A. Delamere, J. Grant, V. Gulick, K. Herkenhoff, L. Keszthelyi, R. Kirk, M. Mellon, S. Squyres, N. Thomas, and C. Weitz, "Mars Reconnaissance Orbiter's High Resolution Imaging Science Experiment (HiRISE)," Journal of Geophysical Research, vol. 112, no. E05S02, doi:10.1029/2005JE002605, 2007.

[6] R. L. Kirk, E. Howington-Kraus, M. R. Rosiek, J. A. Anderson, B. A. Archinal, K. J. Becker, D. A. Cook, D. M. Galuszka, P. E. Geissler, T. M. Hare, I. M. Holmberg, L. P. Keszthelyi, B. L. Redding, W. A. Delamere, D. Gallagher, J. D. Chapel, E. M. Eliason, R. King, and A. S. McEwen, "Ultrahigh resolution topographic mapping of mars with MRO HiRISE stereo images: Meterscale slopes of candidate Phoenix landing sites," Journal of Geophysical Research, vol. 113, no. E00A24, doi:10.1029/2007JE003000, 2008.

[7] J. Hilland, A. M. Bhanji, and P. Estabrook, "Mars Exploration Rover telecommunications subsystem," in Proceedings of the SPIE Microwave and Optical Technology, vol. 5445, April 2004, pp. 38-43. 


\section{BIOGRAPHY}

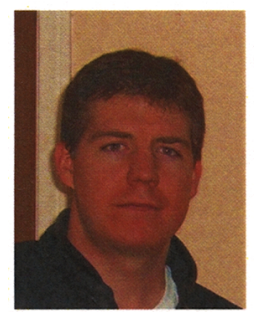

Daniel Gaines is a Mission Manager for the Mars Exploration Rovers mission and a Senior Member of the Artificial Intelligence Group at the Jet Propulsion Laboratory. He received a Ph.D. in Computer Science from the University of Illinois in Urbana Champaign in 1998. Dr. Gaines was an assistant professor of Computer Science at Vanderbilt University prior to joining the Jet Propulsion Laboratory in 2001. In addition to working operations on the MER mission, Dr. Gaines performs research in high level autonomy for in situ robotics. This work includes the development of planning and execution technologies for future in situ robotics missions. His autonomy technology has been demonstrated on prototype rovers, unmanned sea vehicles and an aerobot.

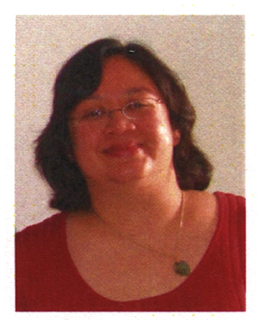

Jennifer Herman is the primary Power subsystem engineer for the Mars Exploration Rover (MER) project at the Jet Propulsion Laboratory (JPL). She received a B.S. in chemistry from the California Institute of Technology and a M.S. in chemical engineering from the University of California at Los Angeles.

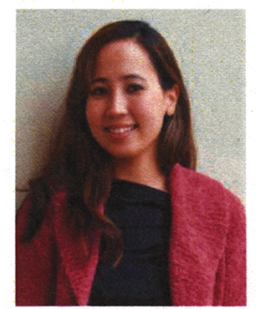

Pauline Hwang is currently the Strategic team lead on the Mars Exploration Rover (MER) project. In addition, she also has Tactical duties on the MER project as an Tactical Uplink Lead, Tactical Activity Planner and Sequence Integration Engineer. She has a B.S. degree from the University of Colorado,

Boulder in Aerospace Engineering and a minor in Astrophysics and has been at NASA's Jet Propulsion Laboratory (JPL) since 2001. Pauline has worked operations on many different JPL missions. After only 4 months at JPL Pauline took over as the Mars Global Surveyor Orbiter sequence lead - She developed and implemented a compensated targeted pointing process that enabled the Mars Orbit Camera (MOC) to return higher resolution images than ever seen on Mars at that time. She helped coordinate and implement MGS relay support of both Spirit and Opportunity's EDL and the surface mission. She first joined the MER project for the launch and cruise phases, Pauline worked operations as a sequence engineer and uplink lead. She later worked on the development of the Mission Operation System of the Dawn Mission and eventually became the Lead Mission Planner for development, launch, and initial checkout phases. She supported Dawn through Launch and Initial Checkout (ICO) Phases as a Mission Planner and ICO lead.

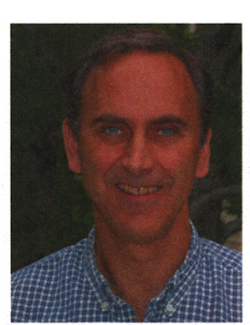

John Grotzinger is a geologist interested in the evolution of surficial environments on Earth and Mars. Field mapping studies are the starting point for more topical laboratory-based studies involving geochemical, geologic, and geochronological techniques. Currently, he is the Project Scientist for the Mars Science Laboratory mission. He is also a member of the Mars Exploration Rover Science Team, and HiRISE team on Mars Reconnaissance Orbiter. He is the Fletcher Jones Professor of Geological Sciences at Caltech.

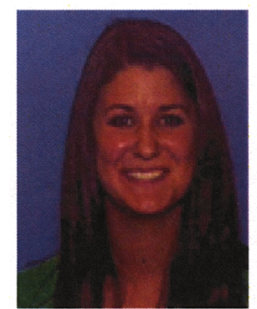

Lauren Edgar is a second year graduate student in the Division of Geological and Planetary Sciences at the California Institute of Technology. She has a B.A. in Earth Sciences modified with Engineering Sciences from Dartmouth College. Under the guidance of John Grotzinger at Caltech, she is studying the geology of Mars through physical sedimentology, stratigraphy and geomorphology. She is also interested in studying potential evidence of early life on Earth and on Mars. Lauren is a Payload Uplink Lead for the Microscopic Imager and Engineering Cameras on the Mars Rovers.

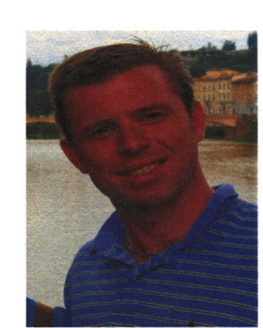

Alex Hayes earned a B.S. in Astronomy and M.Eng in Applied Physics from Cornell University in 2003. Following graduation he represented Cornell at Jet Propulsion Laboratories supporting primary mission operations for the Mars Exploration Rover (MER) spacecraft. Prior to entering graduate school at the California Institute of Technology in fall 2006, he worked for MIT Lincoln Laboratory as technical directory of an infrared seeker test and emulation facility. Currently, he is pursuing a doctoral degree in Planetary Science using Cassini and MER remote sensing data. His area of expertise include terrestrial body remote sensing and spacecraft instrumentation.

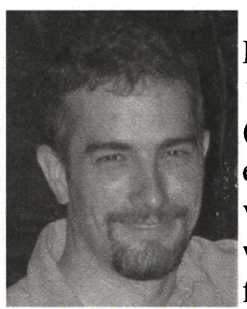

Trent Hare was born in Dover Delaware in 1971. He graduated in 1993 from Northern Arizona University (NAU) with a degree in Computer Mathematics and is currently pursing an advanced degree in engineering. He has worked for the U.S. Geological Survey for nearly 20 years as a GIS and IT specialist, cartographer, and programmer. He has participated on several NASA planetary missions including the Mars Exploration Rovers (MER), High Resolution Imaging Science Experiment (HiRISE), Cassini, and Mars Pathfinder Working Groups. He has published a global and several large country-wide GIS databases for China and Afghanistan. He 
also strives to better support planetary coordinate systems and scientific data sets through open standards by working with the Open Geospatial Consortium (OGC) and several geospatial software initiatives.

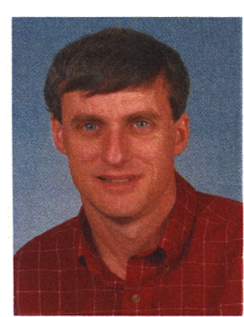

Steve Squyres is Goldwin Smith Professor of Astronomy at Cornell University, and is the Principal Investigator for the science payload on the Mars Exploration Rover Project. His main areas of scientific interest have been Mars and the moons of the outer planets. Dr. Squyres has served as Chair of the NASA Space Science Advisory Committee and as a member of the NASA Advisory Council. His awards include the Space Science Award of the American Institute of Aeronautics and Astronautics, the American Astronautical Societys Carl Sagan Award, the National Space Societys Wernher von Braun Award, and the Benjamin Franklin Medal of the Franklin Institute. He is a fellow of the American Academy of Arts and Sciences. 